\title{
Composición bioquímica del ostión Hyotissa hyotis (Linnaeus, 1758) durante La Niña 1998-1999 y El Niño 1997-1998 en el Golfo de California
}

\author{
Biochemical composition of the oyster Hyotissa hyotis (Linnaeus, 1758) during \\ La Niña 1998-1999 and El Niño 1997-1998 in the Gulf of California
}

\section{Sonia Rodríguez-Astudillo ${ }^{1}$, Marcial Villalejo-Fuerte ${ }^{1}$, Federico García-Domínguez ${ }^{1}$, Rafael Guerrero-Caballero ${ }^{1}$ y Rodolfo Ramírez-Sevilla ${ }^{1}$}

\author{
${ }^{1}$ Instituto Politécnico Nacional, Centro Interdisciplinario de Ciencias Marinas \\ La Paz, Baja California Sur, México. A.P. 592. C.P. 23000 \\ srodrig@ipn.mx
}

\begin{abstract}
Changes in biochemical composition of adductor muscle, gonad, and digestive gland of Hyotissa hyotis, and their relationship to reproduction were studied during the events of La Niña (1998-1999) and El Niño (19971998). In the last event, adductor muscle was the main storage organ of protein and carbohydrates. Lipids were mainly stored in gonad and digestive gland. Although proteins were the highest concentrated substrate in the three organs studied, they were not utilized in reproduction; therefore proteins accumulate in maturity and their highest values occur at spawning during August. Changes in carbohydrates concentration of adductor muscle suggest that this is the most utilized substrate during the reproductive cycle. When comparing the two events, El Niño and La Niña,
\end{abstract}

a significant difference in concentration of protein and carbohydrates occurs in the last period. It is likely that environment conditions prevailing during this period enhance abundance of protein-rich food, especially in summer and autumn. With regards to the accumulated lipid concentration it is concluded that both events El Niño and La Niña did not affect it. In the two events, the highest gonad lipid concentration was noted during reproduction. As decreasing the adductor muscle carbohydrates, lipids of digestive gland and gonad are used up, thus furnishing the needed energy for the rest of the spawning season.

Key words: Proteins, lipids, carbohydrates, Bivalvia, gonadal index

\section{Introducción}

El ostión Hyotissa hyotis (Linnaeus, 1758) es un recurso pesquero potencial viable; sin embargo, en México ha sido poco estudiado, se ha realizado un estudio de taxonomía (Sevilla et al. 1998), uno del ciclo reproductivo (Duprat-Bertazzi \& GarcíaDomínguez 2005) y otro sobre la alimentación y su relación con la temporada reproductiva (VillalejoFuerte et al. 2005). Otro estudio, sobre la relación entre la reproducción y la composición bioquímica fue realizado por Rodríguez-Astudillo et al. (2005), autores que efectuaron su estudio entre noviembre de 1996 y octubre de 1998, meses que de acuerdo al criterio de García-Cuellar et al. (2004), corresponden a un periodo influenciado por el fenómeno ambiental de El Niño. Con excepción de este último estudio, en bivalvos marinos no se han relacionado los efectos producidos por los cambios de temperatura en las variaciones de la composición bioquímica y su relación con el ciclo reproductivo, debidos a El Niño y La Niña. En contraste, el efecto que causan los cambios ambientales producidos por estos fenómenos, en el ciclo reproductivo de bivalvos marinos ha sido estudiado en varias especies, por ejemplo en Gari solida (Urban \& Tarazona 1996), Tagelus dombeii (Ishiyama \& Shiga 1998), Pinctada mazatlanica (García-Cuellar et al. 2004), Donax dentifer (Riascos 2006) y Argopecten purpuratus (Cantillanez et al. 2005).

En general, en bivalvos marinos, la reproducción esta relacionada con los ciclos de almacenamientoutilización de sustancias de reserva en órganos especializados y factores ambientales como la temperatura del agua y disponibilidad de alimento (Gabbot 1975, Bayne 1976, Claereboudt \& Himmelman 1997, Boadas et al. 1997, Barber \& Blake 
1981, Kang et al. 2000, Rodríguez-Astudillo et al. 2002, 2005). En los organismos juveniles la energía disponible se utiliza para el crecimiento; cuando alcanzan la madurez reproductiva, parte de la energía se utiliza en la producción y crecimiento de los gametos por lo cual, la concentración de sustratos energéticos puede cambiar, de acuerdo a los factores ambientales y a los requerimientos metabólicos (Lodeiros et al. 2001, Ojea et al. 2004). En el presente estudio se analiza la relación en la concentración de proteínas, carbohidratos y lípidos almacenados en la gónada, glándula digestiva y músculo aductor durante el evento de La Niña 1998-1999 en una población silvestre de Hyotissa hyotis, se discute en función del evento reproductivo y se compara con lo sucedido durante El Niño 1997-1998 (Rodríguez-Astudillo et al. 2005) sobre una base estacional.

\section{Material y métodos}

El presente trabajo se realizó con muestras obtenidas en la Isla Espíritu Santo (24 36' 18’N, 110²6’24”W) en el Golfo de California, México, (Fig. 1). Se recolectaron al azar, en promedio, 27 organismos adultos por mes de Hyotissa hyotis mediante buceo autónomo a $10 \mathrm{~m}$ de profundidad entre noviembre de 1998 y octubre de 1999 (periodo de Niña). En cada muestreo se registró la temperatura del agua inmediatamente sobre el banco de organismos mediante un termómetro de cubeta.
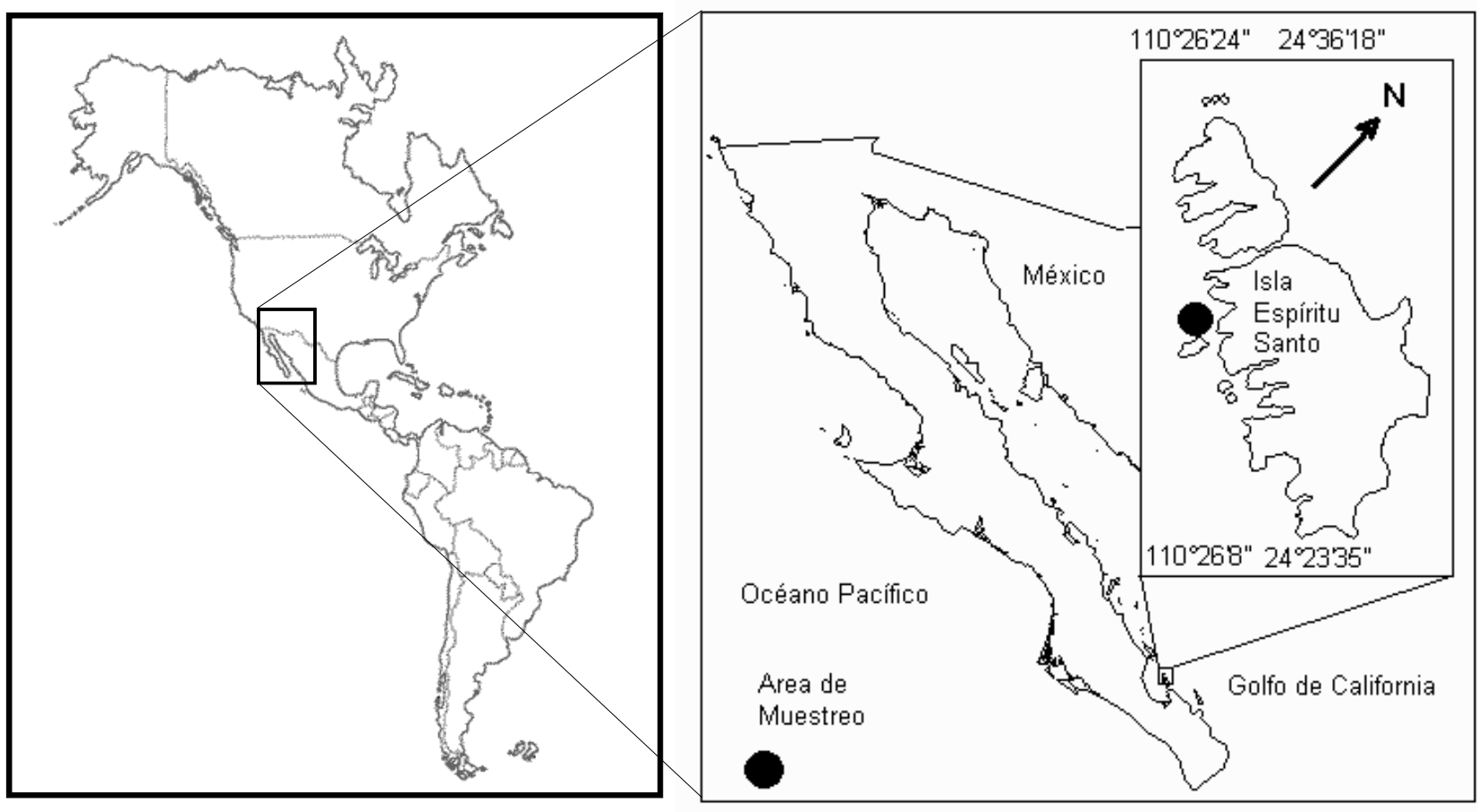

Figura 1

Localización del área de muestreo en la Isla Espíritu Santo, Golfo de California, México

Location of the sampling area at Espiritu Santo Island, Gulf of California, Mexico 


\section{Análisis de composición bioquímica}

Para el análisis bioquímico se siguió el criterio de Rodríguez-Astudillo et al. (2005), que consiste en utilizar 10 organismos por mes. Inmediatamente después de cada muestreo, los ejemplares se llevaron al laboratorio, se limpiaron, se eliminó el agua de la cavidad paleal y se lavaron con agua destilada. Posteriormente, las partes blandas fueron separadas de la concha y pesadas para después llevar a cabo la disección del músculo aductor, gónada y glándula digestiva, cada órgano se pesó y secó en un horno a $100^{\circ} \mathrm{C}$ durante $24 \mathrm{~h}$ (Giese 1967, Ojea et al. 2004, Paez-Osuna et al. 1993).

La determinación de proteínas se llevó a cabo por el método de Lowry et al. (1951). Los carbohidratos se determinaron por el método de Hewitt (1958). La extracción de los lípidos se llevó a cabo con el método de Folch et al. (1957) y una modificación de Bligh \& Dyer (1959).

\section{Temporada reproductiva}

Para relacionar la composición bioquímica con el evento reproductivo se estimó la temporada de reproducción; para ello, se calculó el índice gonádico usando el criterio de Sastry \& Blake (1970), el cual consiste en dividir el peso de la gónada entre el peso del organismo sin concha expresado en porcentaje; los cálculos se realizaron utilizando 27 organismos por mes. La curva resultante del índice gonádico se interpretó de acuerdo a Villalejo-Fuerte \& CeballosVázquez (1996). Los valores más bajos fueron considerados como evidencia de inactividad gametogénica o postdesove, mientras que un aumento sostenido en el índice gonádico se consideró un indicador de actividad gametogénica. Los puntos máximos en la curva fueron considerados como evidencia de madurez y una disminución sostenida fue considerada como evidencia de que la población estaba desovando. Durante los muestreos se registró la temperatura del agua al momento de las recolectas.

\section{Análisis de correlación}

Mediante un análisis de correlación de Spearman se determinaron las correlaciones entre las concentraciones de carbohidratos, lípidos, proteínas, índice gonádico y temperatura. Para la aplicación de este análisis se hicieron las transformaciones correspondientes para obtener la normalidad y homoscedasticidad de los datos (Sokal \& Rohlf 1995). Este análisis se interpretó en forma mensual con respecto al periodo Niña.

\section{Comparación de los periodos Niño-Niña}

Para la comparación con el periodo Niño 1997-1998, se utilizaron los datos que publicamos previamente, y que fueron obtenidos mediante la misma metodología (Rodríguez-Astudillo et al. 2005).

Para dicha comparación los datos correspondientes a cada mes fueron agregados por estaciones del año (Tablas 1-3), lo que permitió hacer un análisis de varianza multifactorial con réplicas para cada uno de los componentes bioquímicos, siendo los factores, el periodo (Niño y Niña), el tejido u órgano en que se hizo la determinación (músculo, gónada o glándula digestiva) y la estación del año, donde se consideró que el invierno estaba conformado por los meses de diciembre a febrero, y así sucesivamente. Debido a que se le dio esta estructura a la información recabada, fue posible determinar la significancia de los factores mencionados, así como la de sus interacciones. El número de réplicas por casilla no fue igual en todos los casos, ya que no fue posible obtener los valores correspondientes a la gónada del mes de diciembre del año Niño, así como los valores propios de abril del año Niña para ninguno de los tejidos.

\section{Análisis de varianza multifactorial}

Para saber si los datos cubrían los requisitos de normalidad y homoscedasticidad exigidos por el análisis multifactorial, se utilizó el programa SigmaStat 3.0. Para los tres componentes bioquímicos se encontró que los datos no seguían una distribución normal $(P<0,001)$, pero cubrían el requisito de ser homoscedásticos. Dado que ninguna transformación permitió que los valores obtenidos se normalizaran, se procedió a realizar el análisis de varianza multifactorial de cada familia química aplicando el programa Statistica 6.0 a los valores sin transformar. La decisión de realizar el análisis multifactorial, pese a que no se cumplía el requisito de normalidad, se debió a que no existen pruebas no paramétricas equivalentes, y a que el análisis de varianza se considera robusto en cuanto a desviaciones a la normalidad (Sokal \& Rohlf 1995). 
Tabla 1

Valores promedio, desviación estándar (DE) y número de datos (n) para carbohidratos de $H$. hyotis

Mean values, standard deviation (SD) and number of data (n) for carbohydrates in H. hyotis

\begin{tabular}{lllllll}
\hline \multirow{2}{*}{ Periodo } & Órgano & Estadístico & Invierno & Primavera & Verano & Otoño \\
\hline \multirow{2}{*}{ Niño (Rodríguez-Astudillo et al. 2005) } & Músculo & Media & 30,63 & 29,67 & 32,89 & 29,25 \\
& & DE (n) & $11,03(3)$ & $10,10(3)$ & $11,49(3)$ & $10,79(3)$ \\
& Gónada & Media & 25,82 & 19,13 & 30,24 & 24,16 \\
& & DE (n) & $6,21(2)$ & $5,29(3)$ & $5,31(3)$ & $9,76(3)$ \\
& Glándula & Media & 25,28 & 21,80 & 22,95 & 20,21 \\
& digestiva & DE (n) & $3,10(3)$ & $2,27(3)$ & $4,99(3)$ & $12,41(3)$ \\
\hline Niña (Este estudio) & Músculo & Media & 54,69 & 60,24 & 49,34 & 38,21 \\
& & DE (n) & $7,50(3)$ & $3,49(2)$ & $13,08(3)$ & $2,29(3)$ \\
& Gónada & Media & 23,33 & 20,93 & 26,19 & 27,04 \\
& & DE (n) & $1,80(3)$ & $3,37(2)$ & $1,85(3)$ & $2,95(3)$ \\
& Glándula & Media & 20,99 & 24,07 & 24,27 & 21,00 \\
& digestiva & DE (n) & $0,93(3)$ & $3,06(2)$ & $2,07(3)$ & $2,17(3)$ \\
\hline
\end{tabular}

Tabla 2

Valores promedio, desviación estándar (DE) y número de datos (n) para proteínas de $H$. hyotis

Mean values, standard deviation (SD) and number of data (n) for proteins in H. hyotis

\begin{tabular}{lllllll}
\hline \multirow{2}{*}{ Periodo } & Órgano & Estadístico & Invierno & Primavera & Verano & Otoño \\
\hline \multirow{2}{*}{ Niño (Rodríguez-Astudillo et al. 2005) } & Músculo & Media & 381,82 & 321,80 & 190,97 & 367,00 \\
& & DE (n) & $67,11(3)$ & $140,37(3)$ & $28,15(3)$ & $41,60(3)$ \\
& Gónada & Media & 422,41 & 214,29 & 212,99 & 324,82 \\
& & DE (n) & $254,64(2)$ & $57,95(3)$ & $71,34(3)$ & $18,33(3)$ \\
& Glándula & Media & 507,03 & 192,27 & 291,58 & 326,55 \\
& digestiva & DE (n) & $226,05(3)$ & $29,14(3)$ & $109,66(3)$ & $87,66(3)$ \\
\hline \multirow{2}{*}{ Niña (Este estudio) } & Músculo & Media & 332,59 & 293,52 & 457,38 & 459,11 \\
& & DE (n) & $10,86(3)$ & $94,34(2)$ & $110,65(3)$ & $120,53(3)$ \\
& Gónada & Media & 363,68 & 306,47 & 333,03 & 401,68 \\
& & DE (n) & $62,18(3)$ & $6,41(2)$ & $76,36(3)$ & $56,36(3)$ \\
& Glándula & Media & 387,00 & 311,65 & 504,44 & 515,67 \\
& digestiva & DE (n) & $10,39(3)$ & $66,86(2)$ & $119,02(3)$ & $134,92(3)$ \\
\hline
\end{tabular}


Tabla 3

Valores promedio, desviación estándar (DE) y número de datos (n) para lípidos de $H$. hyotis

Mean values, standard deviation (SD) and number of data (n) for lipids in $H$. hyotis

\begin{tabular}{lllllll}
\hline Periodo & Órgano & Estadístico & Invierno & Primavera & Verano & Otoño \\
\hline \multirow{2}{*}{ Niño (Rodríguez-Astudillo et al. 2005) } & Músculo & Media & 53,00 & 57,00 & 46,67 & 49,00 \\
& & DE (n) & $5,29(3)$ & $22,54(3)$ & $6,66(3)$ & $7,94(3)$ \\
& Gónada & Media & 67,00 & 127,67 & 102,33 & 85,67 \\
& & DE (n) & $9,90(2)$ & $26,58(3)$ & $2,52(3)$ & $12,50(3)$ \\
& Glándula & Media & 85,67 & 84,00 & 73,33 & 71,67 \\
& digestiva & DE (n) & $13,65(3)$ & $22,34(3)$ & $3,06(3)$ & $16,50(3)$ \\
\hline Niña (Este estudio) & Músculo & Media & 49,17 & 30,25 & 49,17 & 54,00 \\
& & DE (n) & $12,98(3)$ & $0,35(2)$ & $12,90(3)$ & $4,50(3)$ \\
& Gónada & Media & 73,17 & 108,25 & 114,00 & 77,00 \\
& & DE (n) & $16,07(3)$ & $9,55(2)$ & $9,18(3)$ & $12,38(3)$ \\
& Glándula & Media & 95,50 & 96,75 & 82,33 & 76,00 \\
& digestiva & DE (n) & $16,30(3)$ & $15,20(2)$ & $15,70(3)$ & $18,50(3)$ \\
\hline
\end{tabular}

\section{Resultados}

\section{Periodo Niña}

Las temperaturas más bajas fueron registradas de enero a marzo $\left(20-21^{\circ} \mathrm{C}\right)$, coincidiendo con la etapa de inactividad reproductiva. La temperatura empezó a elevarse entre mayo y junio $\left(22\right.$ y $\left.24,5^{\circ} \mathrm{C}\right)$ coincidiendo con la etapa de desarrollo de la gónada y madurez, mientras que los valores más altos se presentaron de julio a octubre $\left(27-29^{\circ} \mathrm{C}\right)$ (Fig. 2), cuando la población se encontraba en desove.

\section{Composición bioquímica}

Las proteínas fueron el principal constituyente en los tres órganos analizados. Durante el periodo Niña, las proteínas siguieron el mismo patrón de comportamiento en músculo y glándula digestiva $(\mathrm{R}=0,80, P<0,01)$. De noviembre a marzo los valores se mantuvieron constantes y se incrementaron de mayo a octubre mientras que en la gónada se mantuvieron sin cambios notables (Fig. 3A). De igual manera, hubo una correlación positiva entre la concentración de proteínas del músculo y de la glándula digestiva con la temperatura del agua $(\mathrm{R}=0,73,0,77$, respectivamente $P<0,02)$.

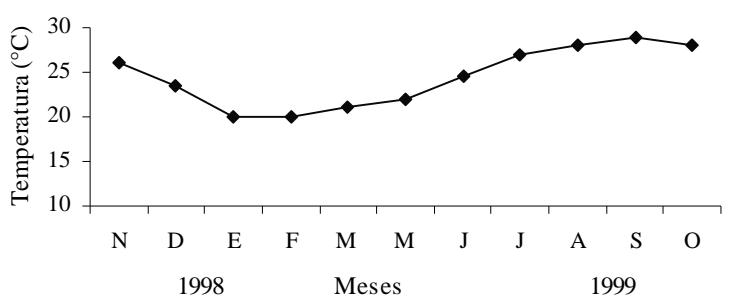

Figura 2

Temperatura superficial del agua en el área de estudio

Surface water temperature in the study area

El músculo aductor fue el principal órgano de almacenamiento de carbohidratos, con valores elevados de diciembre a julio y valores bajos de agosto a noviembre. La gónada y la glándula digestiva no mostraron variación mensual (Fig. 3B). Se encontró una correlación negativa entre los carbohidratos del músculo y los lípidos del músculo $(\mathrm{R}=-0,81, P<0,01)$. 


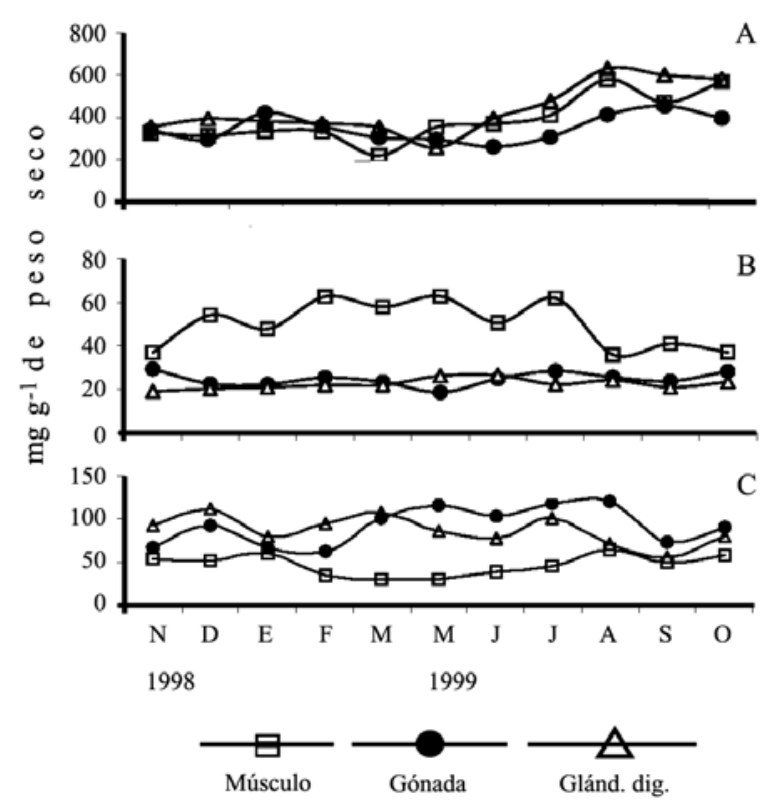

Figura 3

Variación de los componentes bioquímicos en músculo, glándula digestiva y gónada de Hyotissa hyotis. A) Proteínas, B) Carbohidratos y C) Lípidos

Variation of biochemical components in the muscle, digestive gland and gonad of Hyotissa hyotis. A) Protein, B)

Carbohydrate and C) Lipid

Los lípidos en el músculo aductor presentaron menor variación que en los otros tejidos, con valores más bajos en el intervalo de febrero a junio. Los valores de concentración más altos para la gónada se encontraron desde marzo hasta agosto, y los valores más bajos se presentaron en febrero y septiembre. En la glándula digestiva los valores de lípidos se mantuvieron fluctuando durante todo el periodo de estudio; los valores más elevados se presentaron en diciembre, marzo y julio, mientras que los valores bajos ocurrieron en enero, junio y septiembre (Fig. 3C).

\section{Temporada reproductiva}

Se encontraron valores bajos del índice gonádico, que sugieren inactividad reproductiva de noviembre a febrero, la gametogénesis ocurrió entre marzo y mayo mientras que la madurez y el desove se presentaron desde junio hasta septiembre (Fig. 4). Se encontró una correlación positiva entre el índice gonádico y los lípidos de la gónada $(\mathrm{R}=0,80, P<0,01)$.

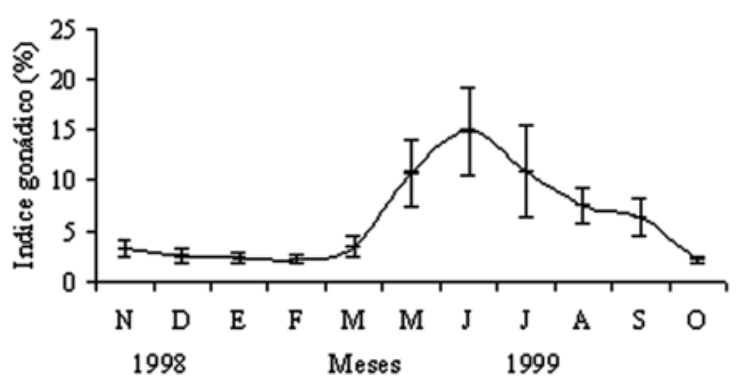

Figura 4

Variación del índice gonádico en Hyotissa hyotis

Variation in gonadal index in Hyotissa hyotis

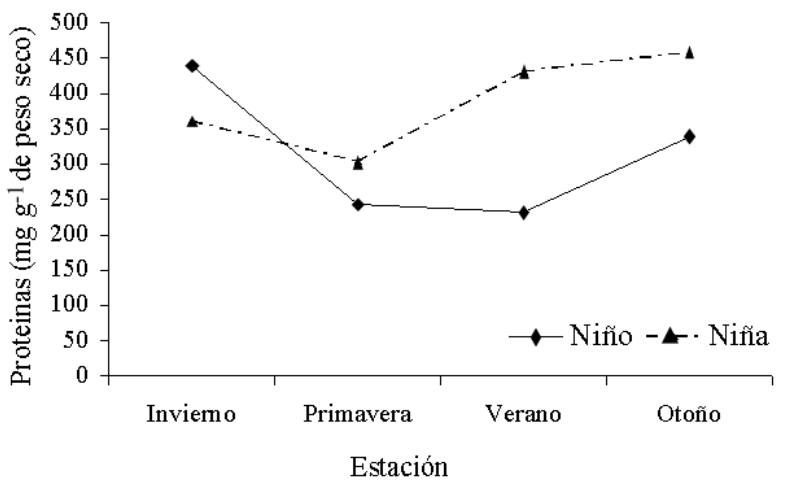

Figura 5

Comparación de la concentración promedio de proteínas en Hyotissa hyotis entre La Niña 1998-1999 y El Niño 1997-1998

Comparison of the mean concentration of proteins in Hyotissa hyotis between La Niña 1998-1999 and El Niño 1997-1998

\section{Comparación del período Niña-Niño}

Respecto a la comparación entre La Niña 1998-1999 y El Niño 1997-1998 encontramos que existió diferencia significativa en la concentración de proteínas acumuladas, siendo mayor durante La Niña. También se encontraron diferencias en la concentración de este sustrato entre las estaciones del año; un hecho interesante fue la interacción significativa entre ambos factores $(P<0,01$ en los tres casos $)$. Esto es consecuencia de los valores superiores para este sustrato observados durante el periodo Niña en verano y otoño (Fig. 5). 


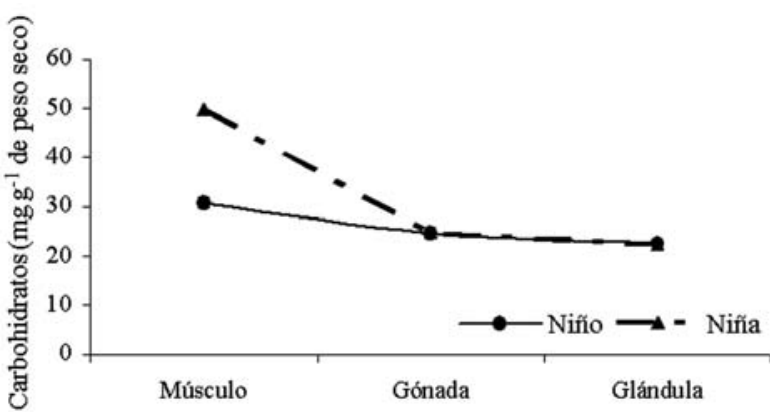

Figura 6

Comparación de la concentración de carbohidratos en Hyotissa hyotis entre La Niña 1998-1999 y El Niño 19971998

Comparison of the mean concentration of carbohydrates in Hyotissa hyotis between La Niña 1998-1999 and El Niño 1997-1998

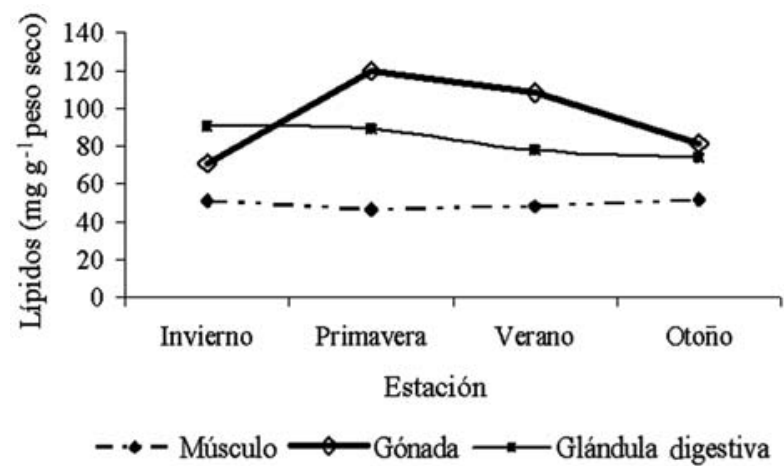

Figura 7

Concentración estacional de lípidos para cada órgano de Hyotissa hyotis

Seasonal lipid concentration for each organ in Hyotissa hyotis

En cuanto a los carbohidratos, en general, se encontró diferencia significativa para los periodos Niño y Niña; de igual manera hubo diferencia significativa para los distintos órganos, así como en la interacción entre ambos factores $(P<0,001)$. Se observó que durante La Niña hubo una acumulación significativamente mayor de carbohidratos en el músculo que durante $\mathrm{El}$ Niño (Fig. 6).

Con respecto a los lípidos se encontró diferencia significativa en la concentración entre los distintos órganos analizados, la gónada tuvo mayor concentración de lípidos que la glándula digestiva y ésta que el músculo $(P<0,001)$, también se encontró diferencia entre las estaciones del año, siendo mayor durante el intervalo primavera-verano $(P<0,05)$. De igual manera se encontró interacción entre ambos factores $(P<0,001)$ (Fig. 7). No se encontraron diferencias significativas en la concentración de lípidos entre El Niño y La Niña.

\section{Discusión}

Aunque las proteínas durante La Niña son el sustrato con concentraciones más elevadas en los tres órganos analizados, no se utilizan durante el periodo reproductivo y, por el contrario, se acumulan durante la madurez, alcanzando los valores más elevados cuando la población se encuentra en desove en agosto. En Crassostrea gigas las proteínas son los componentes principales del músculo y no contribuyen a la producción de gametos los cuales se forman de glucógeno y lípidos almacenados en la glándula digestiva, manto y gónada (Berthelin et al. 2000, Ren et al. 2003).

Los bajos niveles de proteínas registrados durante el periodo de inactividad reproductiva se deben probablemente a que este sustrato es utilizado preferentemente para el crecimiento, el cual ocurre en esta etapa del ciclo reproductivo. Durante la maduración y el desove se utilizan como fuente energética otros componentes bioquímicos de más fácil catabolismo como los carbohidratos almacenados en el músculo aductor y los lípidos de la glándula digestiva y gónada, un resultado similar ha sido informado para los pectínidos Argopecten irradians concentricus (Bayne 1976) y Placopecten magellanicus (Robinson et al. 1981), y para la ostra Crassostrea gigas (Ren et al. 2003).

En la familia Ostreidae la glándula digestiva es el principal órgano relacionado con el almacenamiento de carbohidratos (Matus de La Parra et al. 2005) y en la familia Mytilidae el tejido del manto es el principal órgano de reserva de glucógeno (Matus de La Parra et al. 2005). La tendencia en la curva de concentración de carbohidratos en el músculo aductor sugiere la existencia de un ciclo de almacenamiento de este sustrato que se presenta durante el periodo de inactividad reproductiva y utilización del mismo cuando la población está activa reproductivamente. Un 
resultado similar es reportado en el pectínido Argopecten irradians concentricus (Barber \& Blake 1981).

Parece ser que la etapa de desove requiere una fuerte e inmediata inversión energética, siendo los carbohidratos del músculo aductor quienes soportan este gasto energético. Al disminuir los carbohidratos del músculo aductor, son utilizados los lípidos de la glándula digestiva y de la gónada, quienes aportan la energía necesaria para el resto de la temporada de desove, en ambos casos, la curva de concentración se mantiene con fluctuaciones, siendo más notable la disminución en ambos casos durante el periodo de reproducción; un resultado similar fue encontrado para Aequipecten opercularis (Román et al. 2002).

Respecto a la comparación entre el periodo Niña 1998-1999 y el Niño 1997-1998, la concentración de proteínas acumuladas es mayor en el periodo Niña y la diferencia es significativa, así como entre las estaciones. Es probable que las condiciones ambientales presentes durante el periodo más frío favorezcan la abundancia del alimento rico en proteínas en el medio, sobre todo en verano y otoño.

Se observó durante La Niña una acumulación significativamente mayor de carbohidratos en el músculo que durante El Niño, sin embargo, las variaciones en la temperatura debidas a uno u otro periodo no incidieron en la acumulación de carbohidratos en la gónada ni en la glándula digestiva. Podemos concluir que el músculo aductor es el principal almacén de carbohidratos, independientemente del periodo. De la misma manera que para las proteínas, en el año con menor temperatura promedio, hubo una mayor acumulación de carbohidratos, lo cual sugiere que en años fríos hay mayor cantidad o calidad en el alimento y/o que las temperaturas más bajas facilitan la transferencia de carbohidratos desde el músculo aductor a otros tejidos del organismo.

Con respecto a la diferencia significativa en la concentración de lípidos entre los distintos órganos analizados y la interacción entre los factores, es probable que se deba a que los lípidos se mantienen prácticamente sin cambios en el músculo durante el curso de las estaciones, mientras que en la gónada su concentración es mínima en otoño e invierno y significativamente mayor durante el proceso de maduración gonádica y durante el periodo reproductivo de primavera y verano. La interacción doble entre esos factores también se manifiesta en invierno con la mayor cantidad de lípidos en la glándula digestiva.

En un estudio de $H$. hyotis se encontró que los valores crecientes en la abundancia de fitoplancton en el medio coincidían con el periodo de desarrollo de la gónada y con el cambio estacional de temperatura; por su parte, las diatomeas representaron el 86,5\% y los dinoflagelados el 13,5\% del contenido estomacal (Villalejo-Fuerte et al. 2005), por lo que posiblemente las diatomeas sean las que aportan la mayor parte de las sustancias energéticas que esta especie utiliza en las distintas etapas del evento reproductivo.

Resulta muy interesante que en el caso de los lípidos no se manifiesten diferencias significativas entre los periodos Niño y Niña, lo que parece indicar que el anabolismo y catabolismo de los lípidos depende de rutas metabólicas conservativas, menos influenciables por variaciones interanuales, pero sensibles a los cambios de estación por la existencia de ritmos endógenos circa anuales. La correlación significativa entre los lípidos en la gónada y el índice gonádico parece ser el reflejo de la importancia de los primeros en el ciclo reproductivo en $H$. hyotis.

\section{Agradecimientos}

Al Instituto Politécnico Nacional (IPN) por proporcionar los fondos para la realización del presente trabajo (Proyecto 20070625), a la Comisión de Operación y Fomento de Actividades Académicas del IPN por las becas a S. Rodríguez-Astudillo, F. GarcíaDomínguez, M. Villalejo-Fuerte y R. Ramírez-Sevilla. A los revisores anónimos por sus comentarios $\mathrm{y}$ sugerencias.

\section{Literatura citada}

Barber JB \& NJ Blake. 1981. Energy storage and utilization in relation to gametogenesis in Argopecten irradians concentricus (Say). Journal of Experimental Marine Biology and Ecology 52: 121-134.

Bayne BL. 1976. Aspects of reproduction in bivalves mollusks. En: Wiley ML (ed), Estuarine Processes, pp. 432-448. Academic Press, Nueva York.

Berthelin C, K Kellner \& M Mathieu. 2000. Storage metabolism in the Pacific oyster (Crassostrea gigas) in relation to summer mortalities and reproductive cycle (West coast of France). Comparative Biochemistry and Physiology. Part B, Biochemistry \& Molecular Biology 125: 359-369. 
Bligh EG \& WJ Dyer. 1959. A rapid method of total lipid extraction and purification. Canadian Journal of Biochemical Physiology 37: 911-917.

Boadas MA, O Nusetti, F Mundarain, C Lodeiros \& HE Guderley. 1997. Seasonal variation in the properties of muscle mitochondria from tropical scallop, Euvola (Pecten) ziczac. Marine Biology 128: 247-255.

Cantillanez M, M Avendaño, G Thouzeau \& M Le Pennec. 2005. Reproductive cycle of Argopecten purpuratus (Bivalvia: Pectinidae) in La Rinconada Marine Reserve (Antofagasta, Chile): Response to environmental effects of $\mathrm{El}$ Niño and La Niña. Aquaculture 246: 181-195.

Claereboudt M \& JH Himmelman. 1997. Recruitment, growth and production of giant scallops (Placopecten magellanicus) in Baie des Chaleurs, eastern Canada. Marine Biology 124: 661-670.

Duprat-Bertazzi G \& F García-Domínguez. 2005. Reproductive cycle of the rock oyster Hyotissa hyotis (Linné, 1758) (Griphaeidae) at the La Ballena Island, Gulf of California, Mexico. Journal of Shellfish Research 24: 987-993.

Folch J, M Lees \& GH Sloane-Stanley. 1957. A simple method for the isolation and purification of total lipids from animal tissues. Journal of Biological Chemistry 226: 497-509.

Gabbott PA. 1975. Storage cycles in marine bivalve mollusks: A hypothesis concerning the relationship between glycogen metabolism and gametogenesis. En: Barnes H (ed). Proceedings of the Nineteenth European Marine Biology Symposium, pp. 191-211. Aberdeen University Press, Aberdeen.

García-Cuellar JA, F García-Domínguez, D Lluch-Belda \& S Hernández-Vázquez. 2004. El Niño and La Niña effects on reproductive cycle of the pearl oyster Pinctada mazatlanica (Hanley, 1856) (Pteriidae) at Isla Espiritu Santo in the Gulf of California. Journal of Shellfish Research 23: 113-120.

Giese AC. 1967. Some methods for study of the biochemical constitution of marine invertebrates. Oceanography and Marine Biology an Annual Review 5: 159-186.

Hewitt BR. 1958. Spectrophotometric determination of total carbohydrate. Nature 182(4532): 246-247.

Ishiyama V \& B Shiga. 1998. El fenómeno El Niño 199293: Su influencia en la biología reproductiva de Tagelus dombeii (Mollusca, Bivalvia). Revista Peruana de Biología 5: 37-48.

Kang CK, MS Park, PY Lee, WJ Chol \& WH Lee. 2000. Seasonal variations in condition, reproductive activity, and biochemical composition of the pacific oyster, Crassostrea gigas (Thunberg), in suspended culture in two coastal bays of Korea. Journal of Shellfish Research 19: 771-778.

Lodeiros CJ, JJ Rengel, HE Guderly, O Nusetti \& JH Himmelman. 2001. Biochemical composition and energy allocation in the tropical scallop Lyropecten (Nodipecten) nodosus during the months leading up to and following the development of gonads. Aquaculture 199: 63-72.

Lowry OH, NJ Rosenbrough, AL Farr \& RJ Randall. 1951. Protein measurements with the Folin phenol reagent. Journal of Biological Chemistry 193: 265-275.

Matus de la Parra A, O Garcia \& F San Juan. 2005. Seasonal variations on the biochemical composition and lipid classes of the gonadal and storage tissues of Crassostrea gigas (Thunberg, 1974) in relation to the gametogenic cycle. Journal of Shellfish Research 24: 457-467.

Ojea J, AJ Pazos, D Martinez, S Novoa, JL Sanchez \& M Abad. 2004. Seasonal variation in weight and biochemical composition of the tissues of Ruditapes decussates in relation to the gametogenic cycle. Aquaculture 238: 451-468.

Paez-Osuna F, HM Zazueta-Padilla \& JI Osuna-Lopez. 1993. Biochemical composition of the oysters Crasssostrea iridescens Hanley and Crasssostrea corteziensis Hertlein in the Northwest coast of Mexico: seasonal changes. Journal of Experimental Marine Biology and Ecology 170: 1-9.

Ren JS, ID Marsden, AH Ross \& DR Schiel. 2003. Seasonal variation in the reproductive activity and biochemical composition of the Pacific oyster (Crassostrea gigas) from the Marlborough Sounds, New Zealand. New Zealand Journal of Marine and Freshwater Research 37: 171-182.

Riascos VJM. 2006. Effects of El Niño-Southern oscillation on the population dynamics of the tropical bivalve Donax dentifer from Malaga bay, Colombian Pacific. Marine Biology 148: 1283-1293.

Robinson WE, WE Wehiling, MP Morse \& GC McLeod. 1981. Seasonal changes in soft-body component indices and energy reserves in the Atlantic deep-sea scallop, Placopecten magellanicus. Fishery Bulletin 79: 449-458.

Rodríguez-Astudillo S, M Villalejo-Fuerte, F GarcíaDomínguez \& R Guerrero-Caballero. 2002. Biochemical composition of Spondylus leucacanthus Broderip, 1833 (Bivalvia: Spondylidae) and its relationship with the reproductive cycle at Isla Danzante, Gulf of California, México. Journal of Shellfish Research 21: 757-762 
Rodríguez-Astudillo S, M Villalejo-Fuerte, F GarcíaDomínguez \& R Guerrero-Caballero. 2005. Biochemical composition and its relationship with the gonadal index of the black oyster Hyotissa hyotis (Linnaeus, 1758) at Espiritu Santo Gulf of California. Journal of Shellfish Research 24: 975-978.

Roman G, MJ Campos, J Cano, C Acosta, P Iglesias \& O Garcia. 2002. Reproductive and reserve storage cycles in Aequipecten opercularis (L., 1758) in Galicia, NW Spain. Journal of Shellfish Research 21: 577-584.

Sastry AN \& NJ Blake. 1970. Regulation of gonad development in the bay scallop, Aequipecten irradians Lamark. The Biological Bulletin 140: 274-283.

Sevilla-H M, F Garcia-D \& E Uria-G. 1998. Datos anatómicos de Hyotissa hyotis (Linnaeus, 1758), Ostreacea: Gryphaeidae. Anales de la Escuela Nacional de Ciencias Biológicas 43: 25-32.
Sokal RR \& FJ Rohlf. 1995. Biometría. Principios y métodos estadísticos en la investigación biológica, 832 pp. H. Blume Ediciones, Madrid.

Urban JH \& J Tarazona. 1996. Effects of El Niño/Southern Oscillation on the population dynamics of a Gari solida population (Bivalvia: Psammobidae) from Bahia Independencia, Peru. Marine Biology 125: 725-734.

Villalejo-Fuerte M \& BP Ceballos-Vázquez. 1996. Variación de los índices de condición general, gonádico y de rendimiento muscular en Argopecten circularis (Bivalvia: Pectinidae). Revista de Biología Tropical 44: 591-594.

Villalejo-Fuerte M, MS Muñetón-Gómez \& I GárateLizárraga. 2005. Gut content, phytoplankton abundance and reproductive season in the black oyster (Hyotissa hyotis, Linné, 1758) at Isla Espiritu Santo, Gulf of California. Journal of Shellfish Research 24: 185-190.

Recibido el 5 de abril de 2007 y aceptado el 3 de septiembre de 2007 